\title{
Novel Drug Delivery Device Using Silicone: Controlled Release of Insoluble Drugs or Two Kinds of Water-Soluble Drugs
}

\author{
Masako Kajihara, ${ }^{*, a}$ Toshihiko Sugie, ${ }^{a}$ Hiroo Maeda, ${ }^{a}$ Akihiko Sano, ${ }^{a}$ Keiji FujIoKa, ${ }^{a}$ \\ Yosuke URABE, ${ }^{b}$ Masao TANIHARA, ${ }^{c}$ and Yukio IMANISHI ${ }^{c}$ \\ ${ }^{a}$ Research Division, Sumitomo Pharmaceuticals Co., Ltd.; 1-3-45 Kurakakiuchi, Ibaraki, Osaka 567-0878, Japan: ${ }^{b}$ Life \\ Sciences Industry, Dow Corning Asia Ltd.; 8th Fl. AIG Bldg., 1-1-3 Marunouchi, Chiyoda-ku, Tokyo 100-0005, Japan: \\ and ${ }^{c}$ The Graduate School of Materials Science, Nara Institute of Science and Technology; 8916-5 Takayama, Ikoma, \\ Nara 630-0101, Japan. Received May 15, 2002; accepted October 10, 2002
}

\begin{abstract}
Drug release mechanism from silicone carrier differs depending on physicochemical properties of the drug. So far, there have been few reports on controlled release of insoluble drug and on simultaneous release of two kinds of water-soluble drugs. The purposes of this study are to establish methods for (1) continuous release of insoluble drug, and (2) release of two kinds of water-soluble drugs from silicone carrier. Polystyrene beads (PSTB) and proteins such as interferon (IFN) and human serum albumin (HSA) were used as model drugs. PSTB was released from silicone only when citric acid $(\mathrm{CA})$ and sodium bicarbonate $(\mathrm{SB})$ existed as additives. The release patterns of IFN and HSA were almost same in the case of matrix and covered-rod formulations, but double-layered formulation released them in different patterns. As far as we are aware, this is the first report on the release of insoluble drug from silicone and the controlled release of two kinds of water-soluble drugs.
\end{abstract}

Key words controlled release; silicone; physicochemical property; insoluble drug; water-soluble drug

Aiming at improving drug efficacy, minimizing side effects and promoting patient compliance, extensive studies on drug delivery system (DDS) have been conducted. For example, implantable DDS has been investigated in order to achieve prolonged effects and/or deliver water-soluble drugs which are poorly absorbed by the gastrointestinal tract. Since implantable DDS is administered inside a body, drug carrier materials should be biocompatible and resistant against sterilization. Silicone has been extensively used as a drug carrier material for implantable DDS, because it has appropriate features for such requirements.

Drug release mechanism from silicone carrier differs depending on the physiochemical properties of the drugs as illustrated in Fig. 1. Silicone was originally investigated as carrier materials for lipophilic drugs, and several DDS products have been developed. Although silicone had been believed not to be suitable for delivering water-soluble macromolecules, Hsieh et al. ${ }^{1)}$ and Fujioka et al $^{\left.2-{ }^{2}\right)}$ reported in the 1980 s that proteins could be continuously released from silicone. As described above, a number of studies have been conducted on DDS using silicone. However, investigations have not been perfect to satisfy various medical requirements as pointed out subsequently. First, sustained release of insoluble drugs has been desired. On purpose to develop new vaccine formulations which are effective for a long period of time, sustained release of antigens from silicone carrier has been attempted ${ }^{5}$; viruses or bacteria as antigens are insoluble either in water or in organic solvents. However, sustained release of insoluble drugs from silicone has not been reported so far. Second, simultaneous release of two kinds of watersoluble drugs is often required. For example, simultaneous release of two anti-cancer drugs would bring synergy effect without patients' burden by reducing administration frequency. Interferon (IFN) has anti-cancer and anti-viral effects, and its efficacy is reportedly enhanced by other drugs. ${ }^{6}$ ) In other aspect of vaccine DDS, simultaneous release of antigens and ajuvants is expected to improve its efficacy. Since these drugs are often water-soluble, a method to release two

kinds of water-soluble drugs with suitable release profile should be indispensable.

In this study, we investigated technologies to control the release of drugs with different solubility from silicone carriers. This is the first report on the sustained release of insoluble drugs and the simultaneous release of two kinds of watersoluble drugs from silicone carrier.

\section{Experimental}

Materials Silastic ${ }^{\circledR}$ Q7-4750 Biomedical Grade ETR Elastomer Kit was provided by Dow Corning Corporation (MI, U.S.A.). Q7-4750 is crosslinked by hydrosilylation when Part A and Part B are mixed in equal por-

(a) Lipophilic drugs

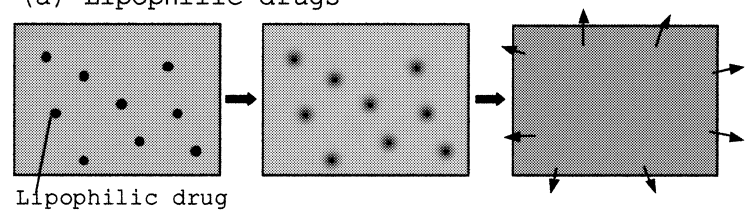

(b) Water-soluble drugs

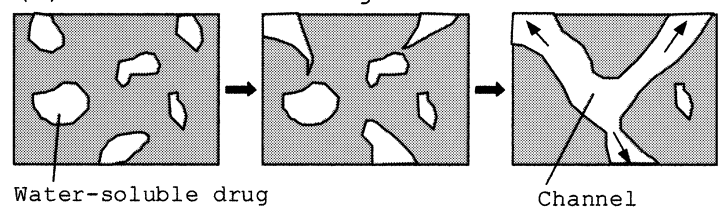

(c) Insoluble drugs

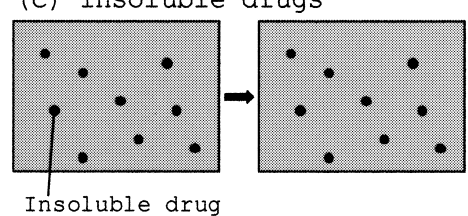

Fig. 1. Release Mechanism of Drugs with Different Physiochemical Properties from a Silicone Carrier

(a) Lipophilic drugs are solubilized in silicone, diffuse, and are released. (b) Watersoluble drugs neither dissolve nor diffuse in silicone. At first, water-soluble drugs existing on the surface area, and then, those existing in the near-surface area dissolve into water. Repetition of these processes leads to channel formation, allowing water-soluble drugs in the bulk to be released. (c) Insoluble drugs neither dissolve nor diffuse in silicone. They are not released because channels are not formed due to insolubility in water. 
tions. Part A consists of dimethylvinylsiloxy-terminated poly(dimethylsiloxane-co-methylvinylsiloxane) and a platinum catalyst. Part B consists of the same siloxane as Part A and a cross-linker.

Citric acid (CA), sodium bicarbonate (SB), glycine (Gly), sodium citrate (SC), mannitol (Man) and sodium azide were purchased from Nacalai Tesque, Inc. (Kyoto, Japan). High-grade gelatin was purchased from Nippi, Inc. (Tokyo, Japan). SC, Man and gelatin were used because they were found to keep IL-1 $\beta$ and IFN stable during lyophilization. As a model for insoluble drugs, polystyrene beads (PSTB) were used. For PSTB, Bacto Latex $^{\circledR} 0.81(0.81 \mu \mathrm{m} \phi)$ and Fluoresbrite ${ }^{\mathbb{B}}$ plain microspheres YG $(1,20$ $\mu \mathrm{m} \phi$ ) were purchased from Difco Laboratories, Inc. (MI, U.S.A.) and PolyScience, Inc. (IL, U.S.A.), respectively. We used human lymphocyte interferon (natural $\alpha$-type interferon, IFN) obtained from Sumitomo Pharmaceuticals Co., Ltd. (Osaka, Japan), and human serum albumin (HSA) Buminate $^{\circledR}$ from Baxter Healthcare Corporation (IL, U.S.A.) as a model of cytokines and water-soluble drugs which bring synergy effect with IFN. IL$1 \beta$ provided by Dr. Lofthouse of the University of Melbourne and avidin (Avi) purchased from Boehringer Mannheim GmbH (Werk Penzberg, Germany) were used as a model of adjuvants and antigens, respectively.

Preparation of Formulations Figure 2 shows the structure of the silicone formulations, and Table 1 lists their compositions.

Preparation of PSTB Silicone Formulations Bacto Latex ${ }^{\circledR} 0.81$ was used for the preparation of PSTB-1 and 2, and Fluoresbrite ${ }^{\circledR}$ was used for the preparation of other formulations. For example, PSTB-6 was prepared as follows. PSTB (1- $\mu \mathrm{m}$ diameter) was washed with water, filtered using a $0.22-\mu \mathrm{m}$ membrane filter and dried under vacuum. This PSTB $(60 \mathrm{mg})$ was

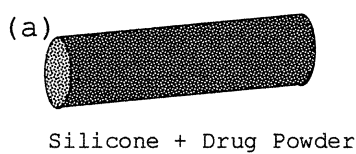

(b)

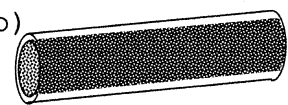

Outer Layer: Silicone

Inner Layer: Silicone + Drug Powder

(C)

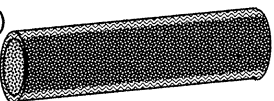

Outer Layer: Silicone + Drug Powder

Inner Layer: Silicone + Drug Powder

(Drug in outer layer is different

from that in inner layer)

Fig. 2. Silicone Formulation

(a) Matrix formulation, (b) covered-rod formulation, (c) double-layered formulation. then mixed with $3.63 \mathrm{~g}$ of CA solution $(100 \mathrm{mg} / \mathrm{ml})$ and freeze dried. The freeze-dried PSTB/CA mixture was passed through a sieve with a $300-\mu \mathrm{m}$ mesh to obtain PSTB/CA powder of the diameter less than $300 \mu \mathrm{m}$. In the same way, SB powder was prepared. After mixing $0.70 \mathrm{~g}$ each of Q7-4750 Part A and Part B, PSTB/CA powder $(282.25 \mathrm{mg})$ and SB powder $(317.75$ $\mathrm{mg}$ ) were added and homogeneously mixed. The mixture was filled into a syringe, extruded through a 1.6 -mm-diameter nozzle, and cured at $37^{\circ} \mathrm{C}$ for $1 \mathrm{~d}$. The cured mixture was cut into 10-mm-long pieces.

Preparation of Double-Layered Silicone Formulations The preparative methods for IFN/HSA-1 (matrix type) and IFN/HSA-2 (covered-rod type) have been reported previously. ${ }^{3,4)}$ For example, double-layered formulation IFN/HSA-3 was prepared as follows. An aqueous solution $(28.2 \mathrm{~g})$ of IFN $(114 \mathrm{MU} / \mathrm{ml})$ was mixed with $2.5 \mathrm{~g}$ of gelatin and freeze-dried. The freeze-dried cake was ground under nitrogen to obtain IFN/gelatin powder. Q7-4750 Part A and Part B (1.05 g each) were mixed with $0.90 \mathrm{~g}$ IFN/gelatin powder and filled into a syringe. Q7-4750 Part A and Part B (17.5 g each) were mixed with $15.0 \mathrm{~g}$ of HSA powder and filled into another syringe. The mixtures were extruded through a concentrically arranged nozzle and die (orifice diameter of 1.6 and $1.9 \mathrm{~mm}$ ) to have IFN/gelatin-containing silicone inside and HSA-containing silicone outside. This was cured at $25^{\circ} \mathrm{C}$ for $3 \mathrm{~d}$. The resultant sample was cut into 10-mm-long pieces.

\section{Evaluation of Drug Release from Formulations}

PSTB Silicone Formulations Formulations were placed in a test tube containing $2 \mathrm{ml}$ of phosphate buffered saline (PBS) (pH 7.4) containing $0.1 \%$ pluronic and $0.01 \%$ sodium azide. The test tube was gently shaken at $37^{\circ} \mathrm{C}$. The amount of PSTB released into PBS during a specified period of time was determined using Fluoroscan II (LabSystems, Co., Finland) with the emission wavelength at $485 \mathrm{~nm}$, and the detection wavelength at $538 \mathrm{~nm}$.

Double-Layered Silicone Formulations Formulations were placed in a test tube containing $2 \mathrm{ml}$ of PBS (pH 7.4) which contains $0.3 \%$ Tween 20 and $0.01 \%$ sodium azide and allowed to stand at $5{ }^{\circ} \mathrm{C}$. At specified periods of time, the amount of IFN released was determined by radioimmunoassay (RIA) and the amounts of HSA, IL- $1 \beta$ and Avi released were determined by enzyme immunoassay (EIA). Since IFN and IL- $1 \beta$ are rather unstable proteins, their stability was confirmed in the buffer during the sampling intervals in advance of the in vitro release study.

\section{Results}

PSTB Silicone Formulations Figure 3a shows the appearance of PSTB-1, 2 and 3 after immersion in PBS for 15 d. PSTB-1 gently swelled and retained its initial shape. PSTB-2 significantly swelled and the surface layer was broken. Change was not observed in PSTB-3. Figure $3 \mathrm{~b}$ shows PSTB-releasing profile of PSTB silicone formulations. No PSTB release was observed from PSTB-3, which does not contain any additives. Similarly, from PSTB-4 containing

Table 1. Composition of Silicone Formulations

\begin{tabular}{|c|c|c|c|c|c|c|}
\hline & \multicolumn{3}{|c|}{ Model drug } & \multicolumn{3}{|c|}{ Formulation } \\
\hline & & Solubility & Content & Type & Size & Additive $^{b)}$ \\
\hline PSTB-1 & $\left.\operatorname{PSTB}(1 \mu \mathrm{m})^{a}\right)$ & \multirow{7}{*}{ Insoluble } & $437 \mu \mathrm{g}$ & Matrix & $2 \phi \times 10$ & Gly $30 \%$ w/w \\
\hline PSTB-2 & $\operatorname{PSTB}(1 \mu \mathrm{m})^{a)}$ & & $469 \mu \mathrm{g}$ & Matrix & $2 \phi \times 10$ & $\mathrm{CA}: \mathrm{SB}=1: 1.5,30 \% \mathrm{w} / \mathrm{w}$ \\
\hline PSTB-3 & $\operatorname{PSTB}(1 \mu \mathrm{m})$ & & $886 \mu \mathrm{g}$ & Matrix & $2 \phi \times 10$ & - \\
\hline PSTB-4 & PSTB $(1 \mu \mathrm{m})$ & & $650 \mu \mathrm{g}$ & Matrix & $2 \phi \times 10$ & Gly $30 \% \mathrm{w} / \mathrm{w}$ \\
\hline PSTB-5 & $\operatorname{PSTB}(1 \mu \mathrm{m})$ & & $508 \mu \mathrm{g}$ & Matrix & $2 \phi \times 10$ & $\mathrm{CA}: \mathrm{SB}=1: 3,15 \% \mathrm{w} / \mathrm{w}$ \\
\hline PSTB-6 & $\operatorname{PSTB}(1 \mu \mathrm{m})$ & & $676 \mu \mathrm{g}$ & Matrix & $2 \phi \times 10$ & $\mathrm{CA}: \mathrm{SB}=1: 3,30 \% \mathrm{w} / \mathrm{w}$ \\
\hline PSTB-7 & $\operatorname{PSTB}(20 \mu \mathrm{m})$ & & $672 \mu \mathrm{g}$ & Matrix & $2 \phi \times 10$ & $\mathrm{CA}: \mathrm{SB}=1: 3,30 \% \mathrm{w} / \mathrm{w}$ \\
\hline \multirow[t]{2}{*}{ IFN/HSA-1 } & IFN & \multirow{8}{*}{ Water-soluble } & $6 \mathrm{MU}$ & Matrix & $2 \phi \times 10$ & Gly $10 \% \mathrm{w} / \mathrm{w}$ \\
\hline & HSA & & $7.8 \mathrm{mg}$ & & & \\
\hline \multirow[t]{2}{*}{ IFN/HSA-2 } & Inside: IFN & & $6 \mathrm{MU}$ & Covered-rod & $2 \phi \times 10$ & Inside: Gly $10 \%$ w/w \\
\hline & Inside: HSA & & $7.2 \mathrm{mg}$ & & & \\
\hline \multirow[t]{2}{*}{ IFN/HSA-3 } & Inside: IFN & & $7 \mathrm{MU}$ & Double-layer & $2 \phi \times 10$ & Inside: Gelatin $30 \% \mathrm{w} / \mathrm{w}$ \\
\hline & Outside: HSA & & $4.6 \mathrm{mg}$ & & & Outside: - \\
\hline \multirow[t]{2}{*}{ IL-1 $\beta /$ Avi-1 } & Inside: Avi & & $868 \mu \mathrm{g}$ & Double-layer & $2 \phi \times 10$ & Inside: $\mathrm{SC}: \mathrm{Man}=1: 2,30 \% \mathrm{w} / \mathrm{w}$ \\
\hline & Outside: IL- $1 \beta$ & & $17 \mu \mathrm{g}$ & & & Outside: $\mathrm{SC}: \mathrm{Man}=1: 2,30 \% \mathrm{w} / \mathrm{w}$ \\
\hline
\end{tabular}

a) PSTB; not fluorescent. b) Additive; kind of additives and their content in the formulations. 

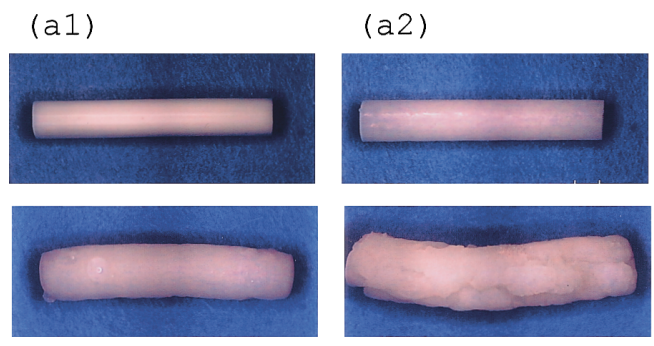

(b)
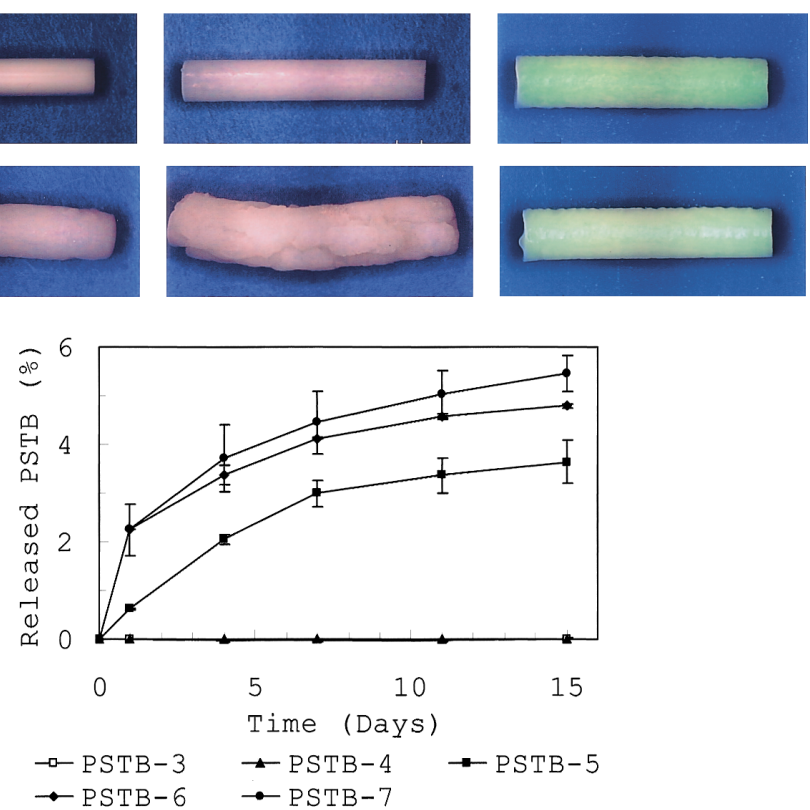

Fig. 3. (a) Appearance of PSTB Silicone Formulations; Top, Initial, Bottom, after 15-d Immersion in PBS; (a1) PSTB-1, (a2) PSTB-2, (a3) PSTB-3, (b) Release of PSTB from Silicone Formulations (Average for $n=2$ )

$30 \% \mathrm{w} / \mathrm{w}$ Gly, no PSTB release was observed. In contrast, PSTB release was observed from PSTB-5, 6 and 7 in which PSTB/CA powder and SB powder were distributed. The amount of released PSTB from PSTB-6 and 7, both containing $30 \% \mathrm{w} / \mathrm{w}$ PSTB/CA and SB powders, was larger than that from PSTB-5, containing $15 \% \mathrm{w} / \mathrm{w}$ PSTB/CA and SB powders. These results indicate that PSTB can be release from silicone by using $\mathrm{CA}$ and $\mathrm{SB}$ as additives, and its release rate can be controlled by amount of CA and SB incorporated in silicone matrix. The particle size of PSTB $(1 \mu \mathrm{m}$ or $20 \mu \mathrm{m}$ ) did not affect the amount of PSTB released.

Simultaneous Controlled Release of Two Kinds of Water-Soluble Drugs

Release from Matrix and Covered-Rod Formulations: Figure 4a shows the release of IFN and HSA from IFN/HSA1 (matrix) and IFN/HSA-2 (covered-rod). The drug release from the matrix formulations was much faster than that from the covered-rod formulations. In the matrix formulation, an initial burst release followed by a slow release took place, while in the covered-rod formulations a slow and monotonous release was observed. For both types of formulations, IFN and HSA were released in the same pattern.

Release from Double-Layered Formulations: Figures $4 \mathrm{~b}$ and $4 \mathrm{c}$ show the release of drugs from double-layered IFN/HSA-3 and IL-1 $\beta /$ Avi-1. For both of them, the release of the outer-layer drug showed an initial burst profile. With IFN/HSA-3, HSA in the outer layer was released rapidly and $97 \%$ of HSA was released within $20 \mathrm{~d}$, while IFN in the inner layer was released gradually. With IL- $1 \beta /$ Avi- 1 too, IL- $1 \beta$ in the outer layer was rapidly released within one day, and Avi in the inner layer was released gradually over $15 \mathrm{~d}$.

\section{Discussion}

It has been reported that a superior immunity is obtained by sustained release of Avi as an antigen model from silicone carrier, as compared to injection of an aqueous Avi solution. ${ }^{5,7)}$ Similar effects are expected by sustained release of live vaccines and inactivated vaccines. Live vaccines are viruses or bacteria whose virulence is weakened, and inactivated vaccines are those treated with denaturants such as formalin. They are not soluble in water or in organic solvents. They do not dissolve and diffuse into silicone unlike lipophilic drugs and do not form channels and cracks unlike water-soluble drugs. Consequently, their release from silicone has long been considered to be impossible. Actually, PSTB as a model of insoluble drug was not released from a silicone carrier when it was dispersed in the silicone without additives (PSTB-3, Fig. 3b). In the in vitro release experiment, when PSTB-3 was immersed in a buffer solution, no swelling was observed (Fig. 3a), suggesting that channels and cracks were not formed in the formulation under these conditions. Even in the presence of $30 \% \mathrm{w} / \mathrm{w}$ Gly as an additive (PSTB-4), PSTB was not released. PSTB-1, which contained $30 \% \mathrm{w} / \mathrm{w}$ Gly as in the case of PSTB-4, swelled after immersion into the buffer solution, suggesting the formation of channels and cracks inside the formulation. Since the original particle size of Gly is $300 \mu \mathrm{m}$, PSTB particles having a diameter of $1 \mu \mathrm{m}$ can pass through channels formed by Gly release. The absence of PSTB release indicates an extremely slow diffusion of PSTB in the channel. It is considered that some driving force could accelerate release of insoluble substances. According to this idea, we used SB and CA as additives. Water-soluble SB and CA might form channels and cracks inside the formulation and react upon mixing to generate carbon dioxide in the channels. The gaseous carbon dioxide would promote release of PSTB. This appears to explain the experimental findings that the silicone formulation containing CA and SB released PSTB in the rate depending on the contents of CA and SB (Fig. 3b). PSTB-2 swelled significantly and many cracks are observed on the surface (Fig. 
(a)

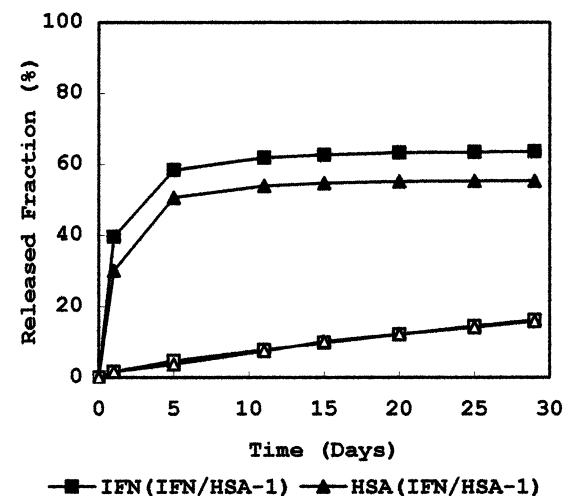

$\rightarrow-$ IFN (IFN/HSA-2) $\triangle-$ HSA (IFN/HSA-2)

(b)

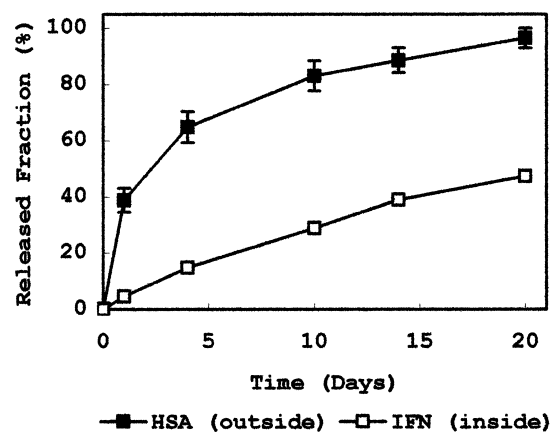

(c)

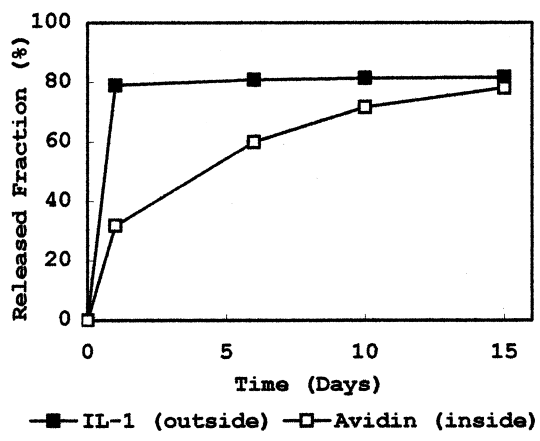

Fig. 4. Release of Drugs from Silicone Formulations Containing Two Kinds of Drugs

(a) Matrix-type IFN/HSA-1 ( $\mathbf{\square}, \mathbf{\Delta}$ ) and covered-rod-type IFN/HSA-2 ( $\square, \triangle$ ) (average for $n=2$ ), (b) double-layered IFN/HSA-3 (average for $n=2$ ), (c) double-layered IL$1 \beta / \operatorname{Avi-1}(n=1)$.

3a), indicating high pressure caused by carbon dioxide gas. Although intense swelling and surface cracks were not observed in PSTB-5, 6 and 7 (observation by naked eyes), these formulations should have released PSTB by the same mechanism. A faster release could be attained by generating a larger amount of carbon dioxide, that is, by changing the kind and content of carbonate and acid.

Depending on the nature of disease, combined use of multiform drugs could yield synergistic effect. ${ }^{6}$ When multiform drugs are released by DDS, the release pattern of each drug should be optimized to obtain the maximum therapeutic effect. In particular, in the case of vaccines, more effective vaccinations would be derived through simultaneous and sustained release of antigen and adjuvant. Since adjuvant often induces strong inflammatory reactions at the administration site, a quick release for a short period is preferable. On the other hand, sustained release of antigens over a long period is desirable to maintain the effect. In order to meet these medical requirements, we developed matrix formulations, covered-rod formulations and double-layered formulations containing two kinds of drugs and investigated their release behaviors. In contrast to covered-rod formulations, water penetrates through an outer layer of double-layered formulations. Therefore, it is expected that the drug in the inner layer would be released in a first-order profile. However, IFN release was more like zero-order kinetics than the first-order kinetics (Fig. 4b). This could have arisen from a slow channel formation in the outer layer, making the inner layer of
IFN/HSA-3 similar to that in a covered-rod formulation. In the case of double-layered formulation of IL-1 $\beta /$ Avi-1 containing SC and Man, which promote drug release, the drug release was faster than that of IFN/HSA-3. Almost all of IL$1 \beta$ existing in the outer layer was released within one day. The release pattern of Avi in the inner layer obeyed the firstorder kinetics (Fig. 4c), supporting the above consideration.

IFN and HSA of IFN/HSA- 1 and IL- $1 \beta$ of IL- $1 \beta /$ Avi- 1 were not completely released from silicone (Figs. 4a, c). This is probably due to insufficient channeling. As shown in Fig. $1 \mathrm{~b}$, water-soluble drugs are released by channeling from silicone. However, some particles of water-soluble drugs dispersed in silicone are isolated and do not form channels. Such particles remain in silicone, because water-soluble drugs do not diffuse in silicone.

It was reported that drug release profiles of matrix and covered-rod formulations were similar between in vitro and in vivo, respectively. ${ }^{4)}$ From this result, drug release profiles of IFN/HSA-1, -2, -3 and IL-1 $\beta$ /Avi-1 in vivo are considered to be similar to those in vitro reported in this paper.

In conclusion, novel methods by which insoluble drugs and two kinds of water-soluble drugs are controlled-released were devised (Fig. 5). For insoluble drugs, by gas generation in the carbonate/acid reaction in silicone carrier achieved the sustained release (Fig. 5a). To release two kinds of water-soluble drugs in the same pattern, powderic drugs should be homogeneously distributed in the formulation. For their release in different patterns, double-layered formulations should be 
(a) Insoluble drugs

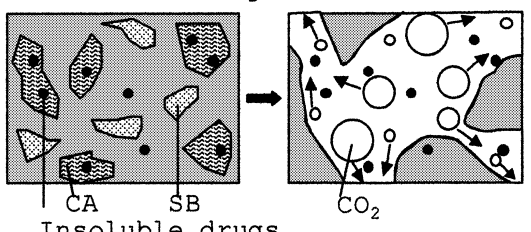

Insoluble drugs

(b) Water-soluble drugs

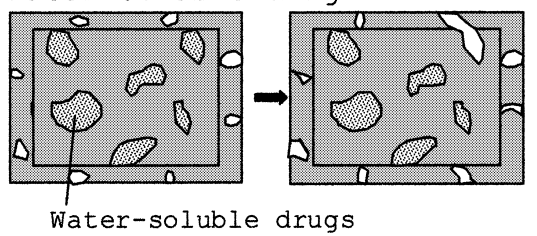

Fig. 5. Diagram Showing the Process of Drug Release from a Silicone Formulation

(a) Insoluble drugs can be released using sodium bicarbonate and citric acid as additives; (b) two kinds of water-soluble drugs can be released with different patterns from double-layered formulations, drug placed in the outer layer is released rapidly, and that in the inner layer slowly. adopted, in which the drug to be released faster is placed in the outer layer, and the drug of slower release in the inner layer (Fig. 5b).

\section{References and Notes}

1) Hsieh D. S. T., Chiang C. C., Desai D. S., Pharm. Tech., 1, 39-49 (1985).

2) Fujioka K., Sato S., Tamura N., Takada Y., Japanese Patent Application Number, JP86-223251.

3) Kajihara M., Sugie T., Mizuno M., Tamura N., Sano A., Fujioka K., Kashiwazaki Y., Yamaoka T., Sugawara S., Urabe Y., J. Control. Release, 66, 49-61 (2000).

4) Kajihara M., Sugie T., Hojo T., Maeda H., Sano A., Fujioka K., Sugawara S., Urabe Y., J. Control. Release, 73, 279-291 (2001).

5) Kemp J. M., Kajihara M., Nagahara S., Sano A., Brandon M., Lofthouse S., Vaccine, 20, 1089-1098 (2002).

6) Furue H., Mugitani H., Hirota F., Suminaga M., Jpn. J. Cancer Chemother, 15, 797-803 (1988).

7) Lofthouse S. A., Kajihara M., Nagahara S., Nash A., Barcham G. J., Sedgmen B., Brandon M. R., Sano A., Vaccine, 20, 1725-1732 (2002). 